\title{
UNILATERAL PROBLEMS FOR ELLIPTIC SYSTEMS WITH GRADIENT CONSTRAINTS
}

\author{
T. N. ROZHKOVSKAYA \\ Institute of Mathematics, Russian Academy of Sciences, Siberian Branch \\ Universitetskǐ Prosp. 4, 630090 Novosibirsk, Russia
}

We consider unilateral problems for elliptic operators $L=\left(L^{1}, \ldots, L^{N}\right)$ with diagonal principal part in a bounded domain $\Omega \subset \mathbb{R}^{n}$. Unilateral problems with gradient constraints have been studied in the scalar case $(N=1)$ for quasilinear elliptic and parabolic operators of general type. The main aim of this article is to extend the corresponding results to systems. The first step is to find an acceptable statement of a unilateral problem with gradient constraints. Comparison of formulations of classical problems and well-known unilateral problems leads to the following conclusion. In both cases we have to find a function $u(x)$ in $\Omega$ using information about the vectors $L u(x) \in \mathbb{R}^{N}, x \in \Omega$. In classical problems we are given values and directions of the vectors $L u(x)$, whereas in unilateral problems only directions at points $x \in \widetilde{\Omega}$, where $\widetilde{\Omega} \subset \Omega$ is not a priori known, are given. In the latter case the direction of $L u(x)$ at $x \in \widetilde{\Omega}$ depends on the value of $u(x)$ (and possibly of $\nabla u(x)$ ).

In the scalar case $(N=1)$ the direction condition is formulated in terms of the sign of $L u(x)$. Such an assumption appears in the obstacle problem and the Evans unilateral problem with gradient constraint. In the obstacle problem for a system the vectors $L u(x) \in \mathbb{R}^{N}$ are directed along the outward normals to given convex sets $K(x) \subset \mathbb{R}^{N}$.

We give the statement of the unilateral problem with constraint on the solution and its gradient. The directions of the vectors $L u(x) \in \mathbb{R}^{N}$ coincide with those of the solution $u(x) \in \mathbb{R}^{N}$ at the points $x \in \Omega$ where $u(x) \neq 0$ and the pair $(u(x), \nabla u(x))$ belongs to the boundary of a given convex set $K(x) \subset \mathbb{R}^{N} \times \mathbb{R}^{N n}$. We give an equivalent statement of the problem in the form of a local quasivariational inequality with some additional conditions.

Using some ideas of the penalty method, we introduce a regularization as a boundary value problem with special penalty. The limit function is the solution 
of the problem considered. This solution satisfies the obstacle problem if we are given the convex constraint only on the solution (not on its gradient).

The paper consists of three sections. In the first some statements of unilateral problems and regularity results are discussed. The statement of the unilateral problem for a system with gradient constraint, and the existence theorems, are formulated in Section 2. A sketch of the proof is given in Section 3. We point out the difficulties which arose in the vector case and refer to earlier articles by the author for some technical details.

Acknowledgement. The author is grateful to Professor W. Zajączkowski for his interest in her earlier results on problems with gradient constraint. The motivation to investigate such problems for systems arose thanks to their discussions.

\section{Preliminaries}

1.1. Regularity of solutions of classical problems. We recall the well-known results of [13]. Let $u_{0}$ be the solution of the Dirichlet problem

$$
L u_{0}=0 \quad \text { in } \Omega, \quad u_{0}=0 \quad \text { on } \partial \Omega
$$

in a bounded domain $\Omega \subset \mathbb{R}^{n}$ for the elliptic system $L=\left(L^{1}, \ldots, L^{N}\right)$, where

$$
L^{r} v=a_{i j}^{r m}(x, v, \nabla v) v_{x_{i} x_{j}}^{m}+a^{r}(x, v, \nabla v) \quad(r=1, \ldots, N),
$$

$v=\left(v^{1}, \ldots, v^{N}\right), \nabla v=\left(v_{x_{1}}^{1}, \ldots, v_{x_{n}}^{1}, \ldots, v_{x_{1}}^{N}, \ldots, v_{x_{n}}^{N}\right)$. In the scalar case $(N=1)$ the differential properties of the solution $u_{0}$ depend on the smoothness of the data and character of non-linearity. In the vector case $(N>1)$ one has to take into account the type of the system. Thus, the regularity result for systems with diagonal principal part is quite similar to that in the scalar case if

$$
a_{i j}^{r}(x, w, p)=a_{i j}(x, w, p) \quad(r=1, \ldots, N),
$$

where $\nu_{1}|\xi|^{2} \leq a_{i j} \xi_{i} \xi_{j}\left(\forall \xi \in \mathbb{R}^{n}\right)$ and the functions $a_{i j}(x, w, p), a^{r}(x, w, p)$ are increasing in $p \in \mathbb{R}^{n N}$ so that

$$
\left|a_{i j}(x, w, p)\right| \leq \mu(|w|)|p|, \quad\left|a^{r}(x, w, p)\right| \leq \mu(|w|)|p|^{2-\delta}, \quad \delta>0,
$$

where $\delta>0, \mu(\tau)$ is a positive continuous function for $\tau>0$. Namely, under the assumptions mentioned, the conditions $a_{i j}, a^{r} \in C^{l+\alpha-2}, \partial \Omega \in C^{l+\alpha}$ with $\alpha>0$ imply $u_{0} \in C^{l+\alpha}$. The analogous results hold for the second and third boundary value problems.

1.2. Regularity of solutions of variational inequalities. Let $L$ be of divergence form, i.e., $L=B$, where

$$
B^{r} v=\frac{d}{d x_{i}} b_{i}^{r}(x, v, \nabla v)+a^{r}(x, v, \nabla v) \quad(r=1, \ldots, N) .
$$

Under some assumptions on $b_{j}^{r}(x, w, p), a^{r}(x, w, p)$ we can define an operator 
$A: \stackrel{\circ}{W}_{m}^{1}\left(\Omega ; \mathbb{R}^{N}\right) \rightarrow W_{m}^{-1}\left(\Omega ; \mathbb{R}^{N}\right)$ by the formula

$$
\langle A \xi, \eta\rangle=\sum_{r=1}^{N} \int_{\Omega}\left[b_{i}^{r}\left(x, \xi, \xi_{x}\right) \eta_{x_{i}}^{r}+a^{r}\left(x, \xi, \xi_{x}\right) \eta^{r}\right] d x .
$$

For $\xi, \eta \in C_{0}^{\infty}\left(\Omega ; \mathbb{R}^{N}\right)$ we have

$$
\langle A \xi, \eta\rangle=-\int_{\Omega} B \xi \cdot \eta d x .
$$

The solution $u_{0}$ of the Dirichlet problem (1.1) satisfies the variational equality

(1.6) find $u_{0} \in \dot{W}_{m}^{1}\left(\Omega ; \mathbb{R}^{N}\right)$ such that

$$
\left\langle A u_{0}, v\right\rangle=0 \quad \text { for all } v \in \stackrel{\circ}{W}_{m}^{1}\left(\Omega ; \mathbb{R}^{N}\right),
$$

which is a special case of the variational inequality

(1.7) find $u \in K$ such that

$$
\langle A u, v-u\rangle \geq 0 \quad \text { for all } v \in K,
$$

where $K$ is a convex closed set in $\stackrel{\circ}{W}_{m}^{1}\left(\Omega ; \mathbb{R}^{N}\right)$.

If the operator $A$ is coercive and pseudomonotone, the classical problem (1.6) has a solution. The latter is considered as a weak solution of the boundary value problem (1.1). It follows from general theorems of convex analysis that the variational inequality (1.7) also has a solution under the same conditions on $A$ and for a convex closed set $K \in \stackrel{\circ}{W}_{m}^{1}\left(\Omega ; \mathbb{R}^{N}\right)$.

However, the regularity questions for variational inequalities differ from those for equations. Indeed, let us take data (i.e., $\left.a_{i j}^{r}, a^{r}, \partial \Omega\right)$ so that $u_{0} \in C_{0}^{\infty}\left(\bar{\Omega} ; \mathbb{R}^{N}\right)$. Take a convex closed set $\mathbf{K} \subset \stackrel{\circ}{m}_{m}^{1}\left(\Omega ; \mathbb{R}^{N}\right)$ and let $u$ be a solution of (1.7) with the same data. For some $\mathbf{K}$ the differential properties of $u$ can be better than those of an arbitrary function in $\stackrel{\circ}{W}_{m}^{1}\left(\Omega ; \mathbb{R}^{N}\right)$, but there exists a function space $W$ such that $C_{0}^{\infty}\left(\bar{\Omega} ; \mathbb{R}^{N}\right) \subset W \subset \stackrel{\circ}{W}_{m}^{1}\left(\Omega ; \mathbb{R}^{N}\right)$, and $u \notin W$. Note that we have such a situation in variational inequalities with pointwise constraints. For example, in the obstacle problem, i.e., the variational inequality (1.7) with the set

$$
\begin{array}{r}
\mathbf{K}=\left\{v \in \stackrel{\circ}{W}_{m}^{1}\left(\Omega ; \mathbb{R}^{N}\right): v(x) \in K(x) \text { a.e. in } \Omega,\right. \\
\left.K(x) \text { is a convex closed set in } \mathbb{R}^{N}\right\},
\end{array}
$$

and in the problem with gradient constraint, i.e. (1.7) with

$$
\begin{array}{r}
\mathbf{K}=\left\{v \in \stackrel{\circ}{W}_{m}^{1}\left(\Omega ; \mathbb{R}^{N}\right): \nabla v(x) \in K(x) \text { a.e. in } \Omega,\right. \\
\left.K(x) \text { is a convex closed set in } \mathbb{R}^{N n}\right\},
\end{array}
$$

we have $u \notin C^{2}\left(\Omega ; \mathbb{R}^{N}\right)$ (see the counterexample in $[15$, Ch. 2, Sec. 8.7]). 
The first result on the regularity of solutions of variational inequalities with sets K of type (1.8) or (1.9) was obtained by H. Lewy and G. Stampacchia in 1969 [14]. Up to now, the scalar case has been studied in detail. The most complete results are established for obstacle problems. Here we mention some regularity results and refer to the books [7, 12] and the survey [25] for precise formulations and bibliography.

In the scalar case $(N=1)$ we have three basic forms of constraints.

(a) The obstacle problem (1.7), (1.8). The limit regularity $\left(u \in W_{\infty}^{2}\right)$ was proved in the case of quasilinear operators. For a sketch of proof see [25].

(b) The thin obstacle problem, i.e., variational inequality of type (1.7), (1.8) but with constraint $u(x) \in K(x)$ for $x \in S$, where $S$ is a given $(n-1)$-dimensional surface in $\bar{\Omega}$, for example $S=\partial \Omega$. The best regularity result (Hölder continuity of the first derivatives) was proved for linear operators in $[5,11,24]$ by different methods.

(c) The problem with gradient constraint. In the case $K(x)=\left\{p \in \mathbb{R}^{n}\right.$ : $|p| \leq 1\}, L v=\Delta v+\mu, \mu=$ const, the variational inequality (1.7), (1.9) is the well-known elastic-plastic torsion problem. The limit regularity $\left(u \in W_{\infty}^{2}\right)$ was apparently proved only in this special case [5]. $W_{q}^{2}$-regularity was established for divergence-type quasilinear operators and some class of strictly convex bounded sets $K(x) \subset \mathbb{R}^{n}[27,17,10]$; see also [7].

Further, the question arises whether the regularity results obtained for the case $N=1$ can be extended to elliptic systems. The answer is affirmative if we consider the obstacle problem for some systems with diagonal principal part. $W_{q}^{2}$-regularity for quasilinear systems was proved by S. Hildebrandt and K.-O. Widman [8]. A. A. Arkhipova and N. N. Ural'tseva have considered the thin obstacle problem [4]. The limit regularity in the obstacle problems for linear diagonal systems with special constraints was also proved; see formulations of results and references in $[25,4,1]$.

The natural regularity result in obstacle problems for a strongly elliptic system is $W_{2}^{2}$-regularity. The corresponding theorems for some modifications of the obstacle problems are given in [25]; see also [2].

The regularity question for the variational inequality for systems with gradient constraint remains open.

1.3. Unilateral problems. The scalar case. The Dirichlet problem is considered both in a weak formulation (1.6) and in a strong form of a boundary value problem (1.1). These statements are formally equivalent for a divergence-type operator $L$.

By analogy, the variational inequality (1.7) can be treated as a weak formulation of a boundary value problem depending on the convex set $\mathbf{K}$. We refer to these boundary problems and their variants as "unilateral problems" and use the term "variational inequality" only for (1.7). We emphasize that these terms are often identified. This does not matter if we restrict ourselves to the obstacle problems with divergence-type operators, since in this case the 
statements are equivalent. However, we have to distinguish between the statements even in the case $N=1, L v=\Delta v+f(x)$ if we deal with gradient constraints.

We begin with the scalar obstacle problem. Let $N=1$ and $u$ be a solution of (1.7), (1.8). Any convex closed set $K(x) \subseteq \mathbb{R}$ can be written in the form $K(x)=\left[\varphi_{1}(x), \varphi_{2}(x)\right]$, where $\varphi_{1}(x) \leq \varphi_{2}(x)$ (the case $\varphi_{1}=-\infty$ or $\varphi_{2}=+\infty$ is admitted). Since $v=\psi w+(1-\psi) u \in \mathbf{K}$ for $\psi \in C_{0}^{\infty}(\Omega), 0 \leq \psi \leq 1$, and $w \in \mathbf{K}$, the variational inequality (1.7), (1.8) is formally equivalent to the pointwise inequality

$$
L u(x) \cdot(w(x)-u(x)) \leq 0 \quad(\forall w \in \mathbf{K})
$$

with the boundary condition $u=0$ on $\partial \Omega$. It follows from (1.10) that a smooth solution $u$ of (1.7), (1.8) satisfies the boundary value problem

$$
\begin{gathered}
\varphi_{1}(x) \leq u(x) \leq \varphi_{2}(x) \quad \text { in } \Omega, \\
L u(x) \begin{cases}\geq 0 & \text { if } u(x)=\varphi_{2}(x), \varphi_{1}(x)<\varphi_{2}(x), \\
=0 & \text { if } \varphi_{1}(x)<u(x)<\varphi_{2}(x), \\
\leq 0 & \text { if } u(x)=\varphi_{1}(x), \varphi_{1}(x)<\varphi_{2}(x),\end{cases} \\
\text { the sign of } L u(x) \text { is arbitrary if } \varphi_{1}(x)=\varphi_{2}(x), \\
u=0 \quad \text { on } \partial \Omega .
\end{gathered}
$$

Note that the sign of $L u(x)$ is not defined for $x \in \Omega$ such that the corresponding set $K(x)$ is degenerate, i.e., int $K(x)=\emptyset$ or $K(x)=\left\{\varphi_{1}(x)\right\}=\left\{\varphi_{2}(x)\right\}$. For every convex set $K(x) \subset \mathbb{R}$ there exists a convex function $G(x, \cdot)$ such that $G(x, \cdot) \in$ $C^{2}(\mathbb{R})$ and

$$
\begin{gathered}
K(x)=\{w \in \mathbb{R}: G(x, w) \leq 0\}=\left[\varphi_{1}(x), \varphi_{2}(x)\right], \\
\operatorname{int} K(x)=\{w \in \mathbb{R}: G(x, w)<0\}=\left(\varphi_{1}(x), \varphi_{2}(x)\right) .
\end{gathered}
$$

Moreover, int $K(x)=\emptyset$ if and only if the value $\varphi_{1}(x)=\varphi_{2}(x)$ is the minimum point of $G(x, \cdot)$. Therefore, in this case $D_{w} G\left(x, \varphi_{1}(x)\right)=0$. Hence we can rewrite (1.11) in terms of $G(x, w)$ as follows:

$$
\begin{gathered}
G(x, u(x)) \leq 0 \quad \text { in } \Omega, \\
L u(x) \uparrow \uparrow \nabla_{w} G(x, u(x)) \quad \text { if } G(x, u(x))=0, D_{w} G(x, u(x)) \neq 0, \\
L u(x)=0 \quad \text { if } G(x, u(x))<0,
\end{gathered}
$$

the sign of $L u(x)$ is not defined if $G(x, u(x))=0$ and $D_{w} G(x, u(x))=0$,

$$
u=0 \quad \text { on } \partial \Omega \text {. }
$$

Here $a \uparrow \uparrow b$ means that either $a=0$ or $a \neq 0$ has the same sign as $b$. If $0 \in K(x)$ $(\forall x \in \bar{\Omega})$, then (1.12) reduces to the following boundary value problem:

$$
\begin{gathered}
u(x) \in K(x), \quad L u(x) \cdot u(x) \geq 0 \quad \text { in } \Omega, \\
L u(x)=0 \quad \text { if } u(x) \in \operatorname{int} K(x), \\
u=0 \quad \text { on } \partial \Omega .
\end{gathered}
$$


Note that the signs of $L u(x)$ and of the solution $u(x)$ have to coincide at $x$ such that $u(x) \in \partial K(x)$. Finally, if $K(x)=\left(-\infty, \varphi_{2}(x)\right]$ and $0 \in K(x)(\forall x \in \bar{\Omega})$, i.e., $\varphi_{2} \geq 0$, we obtain from (1.11) the boundary value problem in the form

$$
\min \{L u(x),-G(x, u(x))\}=0 \quad \text { in } \Omega, \quad u=0 \quad \text { on } \partial \Omega .
$$

Considering (1.11)-(1.14) with a non-divergence-type operator $L$ we lose the connection of the boundary value problems with variational inequalities and in this case the weak solvability does not follow from general results of convex analysis. The obstacle problem with non-divergence-type operators was considered in $[3,23]$, where the solvability in $W_{p}^{2}$ was proved. Note that the existence theorem and the limit regularity $\left(u \in W_{\infty}^{2}\right)$ also follow from the results of [21, 22].

It is easy to write out the corresponding boundary value problems (unilateral problems) for variational inequalities with thin obstacles, boundary obstacles etc. Indeed, in this case the functions $v=\psi u+(1-\psi) w$ are admissible in (1.7) for any $\psi \in C_{0}^{\infty}(\Omega), 0 \leq \psi \leq 1$, and $w \in \mathbf{K}$.

Now we analyze the variational inequality (1.7), (1.9) with gradient constraint. One can show that a solution $u(x)$ of the variational inequality $(1.7),(1.9)$ formally satisfies the conditions

$$
\begin{gathered}
(u(x), \nabla u(x)) \in K(x) \quad \text { in } \Omega, \\
L u(x)=0 \quad \text { if }(u(x), \nabla u(x)) \in \operatorname{int} K(x), \\
u=0 \quad \text { on } \partial \Omega .
\end{gathered}
$$

However, we cannot obtain any information about the sign of $L u(x)$ directly from $(1.7),(1.9)$. It is obvious that the problem (1.15) is not well-posed. L. C. Evans considered the problem (1.15) with an additional condition on the sign of $L u(x)$ in $\Omega$ [6]. Namely, he proved the solvability of the problem (cf. (1.14))

$$
\begin{gathered}
\min \{L u(x),-F(x, u(x), \nabla u(x))\}=0 \quad \text { in } \Omega, \\
u=0 \quad \text { on } \partial \Omega,
\end{gathered}
$$

in the case

$$
\begin{gathered}
L v=a_{i j}(x) v_{x_{i} x_{j}}+a_{i}(x) v_{x_{i}}+a(x) v+f(x), \quad f \geq 0, a \leq 0, \\
F(x, w, p)=|p|^{2}-g(x), \quad g \geq 0,
\end{gathered}
$$

i.e., the sets $K(x)=\left\{p \in \mathbb{R}^{n}:|p|^{2} \leq g(x)\right\}$ are balls in $\mathbb{R}^{n}$ centered at the origin with radius $g(x)$. The assumption $f \geq 0$ in $\Omega$ provides the inequality $u \geq 0$ in $\Omega$. Hence (1.16) implies that $L u(x) \uparrow \uparrow u(x)$ at $x \in \Omega$ such that $F(x, u(x), \nabla u(x))=$ $|\nabla u(x)|^{2}-g(x)=0$. Note that 0 is a minimal value of $u$. Hence $\nabla u(x)=0$ at $x \in \Omega$ such that $u(x)=0$. Consequently, $F(x, u(x), \nabla u(x))<0$ and $L u(x)=0$ on the set of zeros of $u(x)$.

The results of L. C. Evans were generalized in [9, 16, 26, 18-22].

1.4. Unilateral problems. The vector case. We consider the obstacle problem (1.7), (1.8) in the case $N>1$. Let $0 \in K(x)(\forall x \in \Omega)$ and int $K(x)=\emptyset$ if and 
only if $K(x)=\{0\}$; moreover, $\partial K(x) \in C^{1}$ for $x \in \Omega$ such that int $K(x) \neq \emptyset$. It follows that the solution $u$ of (1.7), (1.8) satisfies the pointwise inequality

$$
L u \cdot(v-u) \leq 0 \quad\left(\forall v \in C_{0}^{\infty}(\Omega), v(x) \in K(x)\right) .
$$

For vectors $a \in \mathbb{R}^{N}, b \in \mathbb{R}^{N}$ we shall write $a \uparrow \uparrow b$ if there exists $\lambda \geq 0$ such that $a=\lambda b$. In particular, $a \uparrow \uparrow 0$ means $a=0$. It is easy to see that (1.7), (1.8) is formally equivalent to the next problem:

$$
\begin{gathered}
u(x) \in K(x) \quad \text { in } \Omega, \\
L u(x)=0 \quad \text { if } u(x) \in \operatorname{int} K(x), \operatorname{int} K(x) \neq \emptyset, \\
L u(x) \uparrow \uparrow \mathbf{n}(x, u(x)) \quad \text { if } u(x) \in \partial K(x), \operatorname{int} K(x) \neq \emptyset,
\end{gathered}
$$

the direction of $L u(x)$ is arbitrary if int $K(x)=\emptyset$, i.e., $K(x)=\{0\}$,

$$
u=0 \quad \text { on } \partial \Omega \text {, }
$$

where $\mathbf{n}(x, w)$ denotes the unit outward normal vector to $\partial K(x)$ at $w \in \partial K(x)$. Let $G(x, w)$ be a convex function such that

$$
\begin{gathered}
G(x, 0) \leq 0, \quad G(x, \cdot) \in C^{1}, \\
K(x)=\left\{w \in \mathbb{R}^{N}: G(x, w) \leq 0\right\}, \quad \operatorname{int} K(x)=\left\{w \in \mathbb{R}^{N}: G(x, w)<0\right\},
\end{gathered}
$$

int $K(x)=\emptyset$ if and only if $\nabla_{w} G(x, 0)=0$. To construct $G(x, w)$ one can use the distance function. For example, in the case of balls $K(x)=\left\{w \in \mathbb{R}^{N}:|w|^{2} \leq\right.$ $g(x)\}$ we can take $G(x, w)=|w|^{2}-g(x)$. If $g\left(x_{0}\right)>0$ for $x_{0} \in \bar{\Omega}$ and $|w|^{2}=g\left(x_{0}\right)$, then the vector $\nabla_{w} G(x, w)$ is directed along the outward normal to $\partial K(x)$, i.e., $\mathbf{n}(x, w)=\lambda \nabla_{w} G(x, w)=2 \lambda w$, where $\lambda>0$. Thus, the unilateral problem (1.18) can be written as follows:

$$
\begin{gathered}
G(x, u(x)) \leq 0 \quad \text { in } \Omega, \\
L u(x)=0 \quad \text { if } G(x, u(x))<0, \\
L u(x) \uparrow \uparrow \nabla_{w} G(x, u(x)) \quad \text { if } G(x, u(x))=0, \nabla_{w} G(x, u(x)) \neq 0, \\
\text { the direction of } L u(x) \text { is arbitrary if } \nabla_{w} G(x, u(x))=0, \\
u=0 \quad \text { on } \partial \Omega .
\end{gathered}
$$

2. The main result. In this section we formulate a problem with gradient constraint as a local quasivariational inequality under some additional conditions. We show that its regular solution satisfies a unilateral problem and give formulations of the existence theorems. A sketch of the proof is given in Section 3. First we write out assumptions on data.

2.1. The operator $A$. Let us introduce

$$
\langle A u, v\rangle=\sum_{r=1}^{N} \int_{\Omega}\left(a_{i j}(x) u_{x_{i}}^{r} v_{x_{j}}^{r}+\left(a_{i j}(x)\right)_{x_{j}} u_{x_{i}}^{r} v^{r}-a^{r}(x, u, \nabla u) v^{r}\right) d x,
$$

where $a_{i j} \in C^{2}(\bar{\Omega}), a^{r} \in C^{2}\left(\bar{\Omega} \times \mathbb{R}^{N} \times \mathbb{R}^{N n}\right), \Omega$ is a bounded domain in $\mathbb{R}^{n}$ with 
boundary $\partial \Omega \in C^{3}$. Suppose that the ellipticity condition is satisfied:

$$
\nu_{1}|\xi|^{2} \leq a_{i j}(x) \xi_{i} \xi_{j} \leq \mu_{1}|\xi|^{2} \quad\left(\forall \xi \in \mathbb{R}^{n}\right),
$$

and for $x \in \bar{\Omega},|w|>M, p \in \mathbb{R}^{N n}$

$$
a(x, w, p) \cdot w=\sum_{r=1}^{N} a^{r}(x, w, p) w^{r}<0,
$$

while for $x \in \bar{\Omega},|w| \leq M,|p|>R_{1}$

$$
\left|a^{r}(x, w, p)\right| \leq \mu_{2}(|w|)\left(1+|p|^{1+\alpha_{1}}\right),
$$

where $\nu_{1}, \mu_{1}, M, R_{1}$ denote positive constants, the function $\mu_{2}(\tau), \tau>0$, is strictly positive, and $0<\alpha_{1}<1,1 /\left(1-\alpha_{1}\right)<n$. Then (A1) defines an operator $A$ : $\stackrel{\circ}{W}_{2}^{1}\left(\Omega ; \mathbb{R}^{N}\right) \rightarrow W_{2}^{-1}\left(\Omega ; \mathbb{R}^{N}\right)$. Moreover, assume that for $|p|>R_{1}$

$$
\begin{gathered}
\left|\frac{\partial a^{r}(x, w, p)}{\partial x_{k}}\right|+\left|\frac{\partial a^{r}(x, w, p)}{\partial w^{m}}\right| \leq \mu_{3}(|w|)\left(1+|p|^{1+\alpha_{1}}\right), \\
\left|\frac{\partial a^{r}(x, w, p)}{\partial p_{k}^{l}}\right| \leq \mu_{4}(|w|)\left(1+|p|^{\alpha_{1}}\right)
\end{gathered}
$$

where $1 \leq k \leq n$ and $1 \leq l, m \leq N$, the functions $\mu_{3}(\tau), \mu_{4}(\tau)$ are strictly positive and continuous for $\tau>0$.

2.2. The operator $L$. Define

$$
L v=a_{i j}(x) v_{x_{i} x_{j}}^{r}+a^{r}(x, w, p) .
$$

It is easy to see that the integral identity

$$
\langle A u, v\rangle=-\int_{\Omega} L u \cdot v d x
$$

holds for functions $u \in W_{p}^{2}\left(\Omega ; \mathbb{R}^{N}\right), v \in \stackrel{\circ}{W}_{2}^{1}\left(\Omega ; \mathbb{R}^{N}\right)$.

2.3. The function $G: \bar{\Omega} \times \mathbb{R}^{N} \rightarrow \mathbb{R}$. Define

$$
G(x, w)=B(x) w \cdot w=\sum_{l, m=1}^{N} b^{l m}(x) w^{l} w^{m},
$$

where $B(x)=\left(b^{l m}(x)\right)$ is a symmetric positive-definite matrix,

$$
B(x) \zeta \cdot \zeta \geq \nu_{2}|\zeta|^{2} \quad\left(\forall \zeta \in \mathbb{R}^{N}\right),
$$

where $\nu_{2}=$ const $>0$, with elements $b^{l m} \in C^{2}(\bar{\Omega})$. Note that $G(x, w)$ is nonnegative and strictly convex with respect to $w \in \mathbb{R}^{N}$; moreover,

$$
G(x, w)=0 \Leftrightarrow \nabla_{w} G(x, w)=0 \Leftrightarrow B(x)=0 \Leftrightarrow w=0 .
$$

2.4. The function $F: \bar{\Omega} \times \mathbb{R}^{N} \times \mathbb{R}^{N n} \rightarrow \mathbb{R}$. Set

$$
\begin{gathered}
D=\left\{(x, w, p) \in \bar{\Omega} \times \mathbb{R}^{N} \times \mathbb{R}^{n N}: F(x, w, p)>0\right\}, \\
K(x)=\left\{(w, p) \in \mathbb{R}^{N} \times \mathbb{R}^{N n}: F(x, w, p) \leq 0\right\} .
\end{gathered}
$$


Suppose that

$$
F \in C^{1+\alpha_{2}}\left(\bar{\Omega} \times \mathbb{R}^{N} \times \mathbb{R}^{N n}\right) \cap C^{2}(D),
$$

where $0<\alpha_{2}<1$, and let $F(x, w, p)$ be convex with respect to $(w, p) \in \mathbb{R}^{N} \times \mathbb{R}^{N n}$. Then the set $K(x)$ is convex. Moreover, by the definition of a convex function we have for $x \in \Omega, w \in \mathbb{R}^{N}, p \in \mathbb{R}^{N n}$

$$
\sum_{m=1}^{N} \frac{\partial F(x, w, p)}{\partial w^{m}} w^{m}+\sum_{l=1}^{N} \sum_{k=1}^{n} \frac{\partial F(x, w, p)}{\partial p_{k}^{l}} p_{k}^{l} \geq F(x, w, p)-F(x, 0,0) .
$$

We also suppose that $F(x, w, p)$ is strictly convex on $D$ with respect to $p \in \mathbb{R}^{N n}$, i.e.,

$$
\begin{array}{r}
\sum_{m, l=1}^{N} \frac{\partial^{2} F}{\partial u^{m} \partial u^{l}} \zeta^{m} \zeta^{l}+2 \sum_{m, l=1}^{N} \sum_{k=1}^{n} \frac{\partial^{2} F}{\partial u^{m} \partial p_{k}^{l}} \zeta^{m} \eta_{k}^{l}+\sum_{m, l=1}^{N} \sum_{k, s=1}^{n} \frac{\partial^{2} F}{\partial p_{k}^{l} \partial p_{s}^{m}} \eta_{k}^{l} \eta_{s}^{m} \\
\geq \nu_{2}|\zeta|^{2}+\nu_{3}(|w|)\left[1+|p|^{2}\right]^{\left(\alpha_{2}-1\right) / 2}|\eta|^{2} \quad\left(\forall \zeta \in \mathbb{R}^{N}, \eta \in \mathbb{R}^{n N}\right),
\end{array}
$$

where $(x, w, p) \in D, \zeta=\left(\zeta^{1}, \ldots, \zeta^{N}\right) \in \mathbb{R}^{N}, \nu_{2}=$ const $\geq 0$ and $\nu_{3}(\tau)$ is a strictly positive continuous function for $\tau>0$. Furthermore,

$$
F(x, 0,0)<0 \quad(\forall x \in \bar{\Omega})
$$

and there exists a function $R_{2}(\tau)$ such that for all $x \in \bar{\Omega}$

$$
K(x) \subset\left\{(w, p) \in \mathbb{R}^{N} \times \mathbb{R}^{n N}:|p|<R_{2}(|w|)\right\},
$$

and for $x \in \bar{\Omega}, u \in \mathbb{R}^{N},|p| \geq R_{2}(|w|)$

$$
F(x, w, p) \geq \nu_{4}|p|^{1+\alpha_{2}} .
$$

Moreover, for $(x, w, p) \in D$ the following estimates hold:

$$
\begin{gathered}
F(x, w, p)+\left|\frac{\partial F(x, w, p)}{\partial x_{i}}\right|+\left|\frac{\partial^{2} F(x, w, p)}{\partial x_{i} \partial x_{j}}\right| \leq \mu_{5}(|w|)\left[1+|p|^{1+\alpha_{2}}\right], \\
\left|\frac{\partial^{2} F(x, w, p)}{\partial x_{i} \partial p_{k}^{l}}\right|+\left|\frac{F(x, w, p)}{\partial p_{k}^{l}}\right| \leq \mu_{6}(|w|)\left[1+|p|^{\alpha_{2}}\right], \\
\left|\frac{\partial F(x, w, p)}{\partial w^{m}}\right|+\left|\frac{\partial^{2} F(x, w, p)}{\partial x_{i} \partial w^{m}}\right| \leq \mu_{7}(|w|)\left[1+|p|^{1+\alpha_{2}-\delta}\right],
\end{gathered}
$$

where $1 \leq i, j, k \leq n, 1 \leq l, m \leq N, 0<\alpha_{2}<1, \delta>0$, the functions $\mu_{5}(\tau)$, $\mu_{6}(\tau), \mu_{7}(\tau)$ are continuous and positive for $\tau \geq 0$.

2.5. Formulation of the problem. For $v \in C\left(\Omega ; \mathbb{R}^{N}\right)$ write

$$
\Omega_{v}=\{x \in \Omega: v(x) \neq 0\},
$$

and for $v \in C^{1}\left(\Omega_{v} ; \mathbb{R}^{N}\right)$ set

$$
\Omega_{v F}=\left\{x \in \Omega_{v}: F(x, v(x), \nabla v(x))<0\right\} .
$$


Note that the sets $\Omega_{v}, \Omega_{v F}$ are open in $\Omega$ and the $M(x, v(x))$ are convex closed sets for all $x \in \Omega$. It is obvious that $v(x) \in \partial M(x, v(x))(\forall x \in \Omega)$.

Problem (quasivariational inequality). Find a function

$$
u \in \stackrel{\circ}{W}_{2}^{1}\left(\Omega ; \mathbb{R}^{N}\right) \cap C\left(\Omega ; \mathbb{R}^{N}\right) \cap C^{1}\left(\Omega_{u} ; \mathbb{R}^{N}\right)=V
$$

such that

$$
\langle A u,(v-u) \eta\rangle \geq 0
$$

for all $\eta \in C_{0}^{\infty}(\Omega)$ with $\operatorname{supp} \eta \subset \Omega_{u}, \eta \geq 0$, and all $v \in \stackrel{\circ}{W}_{2}^{1}\left(\Omega ; \mathbb{R}^{N}\right)$ with $v(x) \in M(x, u(x))$ a.e. in $\Omega$,

$$
\begin{gathered}
F(x, u(x), \nabla u(x)) \leq 0 \quad \text { in } \Omega_{u}, \\
\text { (QV4) } \\
A u=0 \quad \text { in } \Omega_{u F} \text { (as distributions). }
\end{gathered}
$$

Problem (unilateral problem). Find a function

$$
u \in C\left(\bar{\Omega} ; \mathbb{R}^{N}\right) \cap W_{p, \text { loc }}^{2}\left(\Omega_{u} ; \mathbb{R}^{N}\right)
$$

such that

$$
\begin{gathered}
F(x, u(x), \nabla u(x)) \leq 0 \quad \text { in } \Omega_{u}, \\
L u(x)=\lambda(x) \nabla_{w} G(x, u(x))=\lambda(x) B(x) u(x) \quad \text { a.e. in } \Omega_{u},
\end{gathered}
$$

where $\lambda(x) \geq 0$ is an a priori unknown function and

$$
\begin{gathered}
\lambda(x)=0 \quad \text { for } x \in \Omega_{u F}, \\
u=0 \quad \text { on } \partial \Omega .
\end{gathered}
$$

Remark 2.1. The problem (U1)-(U5) is a free boundary problem. The domain $\Omega$ is divided into three sets:

$\Omega_{1}: u=0$

$\Omega_{2}: u \neq 0, F(x, u, \nabla u)<0, L u=0$,

$\Omega_{3}: u \neq 0, F(x, u, \nabla u)=0$, either $L u=0$ or $L u(x)$ is directed along the outward normal to $M(x, u(x))$ at $u(x) \in \partial M(x, u(x))$.

Note that the set $M(x, u(x))$ is degenerate for $x \in \Omega_{1}$, i.e., for $x$ such that $M(x, u(x))=\{0\} \Leftrightarrow \operatorname{int} M(x, u(x))=\emptyset$.

R e mark 2.2. The scalar variant of (U1)-(U5) can be written as follows:

$$
\begin{gathered}
F(x, u(x), \nabla u(x)) \leq 0 \quad \text { in } \Omega_{u}, \\
L u(x) \cdot u(x) \geq 0 \quad \text { a.e. in } \Omega, \\
L u(x)=0 \quad \text { in } \Omega_{u F}, \\
u=0 \quad \text { on } \partial \Omega,
\end{gathered}
$$

i.e., the sign of $L u(x) \neq 0$ is the same as that of the solution $u(x)$.

2.6. Proposition. If $u \in C\left(\bar{\Omega} ; \mathbb{R}^{N}\right) \cap W_{p, \text { loc }}^{2}\left(\Omega_{u} ; \mathbb{R}^{N}\right)$ satisfies (QV1)-(QV4), then $u$ is a solution of (U1)-(U5). 
Proof. (U2) is a consequence of (QV3); and (U1), (U5) are valid because of the regularity assumption. To verify (U3) we take $w \in \stackrel{\circ}{W}_{2}^{1}\left(\Omega ; \mathbb{R}^{N}\right)$ such that $w(x) \in M(x, u(x))$ a.e. in $\Omega$ and $\xi \in C_{0}^{\infty}(\Omega)$ with $0 \leq \xi \leq 1$. Then $v=\xi(x) u+$ $(1-\xi(x)) w$ is admissible in (QV2). Hence we can deduce from (QV2) the pointwise inequality

$$
L u(x) \cdot(w(x)-u(x)) \leq 0 \quad \text { a.e. in } \Omega_{u} .
$$

It means that $L u(x)$ either vanishes or else its direction coincides with that of the outward normal $\mathbf{n}(x, u(x))$ to $\partial M(x, w)$ at $w=u(x)$. Since $\mathbf{n}(x, u(x))=$ $\lambda \nabla_{w} G(x, u(x)), \lambda>0$, we obtain (U3). Equality (QV4) yields (U4).

2.7. The penalty problem. Consider the boundary value problem

$$
\begin{gathered}
L u^{\varepsilon}=\beta_{\varepsilon}\left(F\left(x, u^{\varepsilon}, \nabla u^{\varepsilon}\right)\right) B(x) u^{\varepsilon} \quad \text { in } \Omega, \\
u^{\varepsilon}=0 \quad \text { on } \partial \Omega,
\end{gathered}
$$

where $\varepsilon>0, \beta_{\varepsilon}(\tau)=(1 / \varepsilon) \beta_{0}(\tau), \tau \in \mathbb{R}$, and $\beta_{0} \in C^{2}(\mathbb{R})$ satisfies

$$
\begin{gathered}
\beta_{0}(\tau)=0 \quad \text { for } \tau \leq 0, \quad \beta_{0}(\tau)>0 \quad \text { for } \tau>0, \\
\beta_{0}^{\prime}(\tau)>0, \quad \beta_{0}^{\prime \prime}(\tau) \geq 0 \quad \text { for } \tau>0, \\
\beta_{0}(\tau)=\tau^{1+\alpha_{0}} \quad \text { for } \tau \geq 1 .
\end{gathered}
$$

The constant $\alpha_{0}, 0<\alpha_{0}<1$, is such that

$$
\max \left\{0, \frac{\alpha_{1}-\alpha_{2}}{1+\alpha_{2}}\right\}<\alpha_{0}<\frac{1-\alpha_{2}}{1+\alpha_{2}} .
$$

By (F9) we have for $(x, w, p) \in D \cap\{(x, w, p):|w| \leq M\}$

$$
0 \leq \beta_{\varepsilon}(F(x, w, p))=\frac{1}{\varepsilon}[F(x, w, p)]^{1+\alpha_{0}} \leq \frac{1}{\varepsilon} \mu_{5}(M)\left(1+|p|^{\gamma}\right),
$$

where $\gamma=\left(1+\alpha_{0}\right)\left(1+\alpha_{2}\right)$. In view of (PP3) we have $\gamma<2$. Moreover, by (G2),

$$
\beta_{\varepsilon}(F(x, w, p)) B(x) w \cdot w \geq 0 .
$$

From Theorem 5.2 of $\left[13\right.$, Ch. 8] we conclude that there exists a solution $u^{\varepsilon} \in$ $C^{3}\left(\Omega ; \mathbb{R}^{N}\right) \cap C^{1}\left(\bar{\Omega} ; \mathbb{R}^{N}\right)$ of the penalty problem (PP1), (PP2).

2.8. Formulation of the theorems. We assume the conditions from $2.1-2.4$ to be satisfied.

THEOREM 2.1. Suppose that

$$
\nabla_{w} G \cdot \nabla_{w} F+\sum_{l, m=1}^{N} \sum_{k=1}^{n} \frac{\partial F}{\partial p_{k}^{l}} b^{l m} p_{k}^{m} \geq \nu_{5}|p|^{1+\alpha_{2}}-\mu_{7}(|w|) p^{1+\alpha_{2}-\gamma_{2}},
$$

where $\nu_{5}=$ const $>0$ and $\mu_{7}(\tau)$ is a positive continuous function for $\tau \geq 0$. Then there exist a sequence $u^{\varepsilon}$ and a function $u \in V \cap W_{p, \text { loc }}^{2}\left(\Omega_{u} ; \mathbb{R}^{N}\right)$ such that $u^{\varepsilon} \rightarrow u$ in $W_{2}^{-1}\left(\Omega ; \mathbb{R}^{N}\right)$ and $u$ satisfies (QV1)-QV4).

THEOREM 2.2. Let $u$ be the solution of (QV1)-(QV4) from Theorem 2.1. If $B(x)$ is the unit matrix then $u \in C^{0,1}\left(\bar{\Omega} ; \mathbb{R}^{N}\right)$. 
COROLlaRY. Under the assumption of Theorem 2.2 the solution $u$ of (QV1)(QV4) from Theorem 2.1 is a solution of the unilateral problem (U1)-(U5).

If constraint is given only on the solution, then the regularizations introduced above yield the solution of the obstacle problem. We show this in a simple case and refer to the article of S. Hildebrandt and K.-O. Widman [8] for regularity results in the general case. Let

$$
\begin{gathered}
L v=\Delta v^{l}+a^{l m}(x) w^{m}+a^{l}(x) \quad(l=1, \ldots, N), \\
G(x, w)=|w|^{2}, \\
F(x, w, p)=|w|^{2}-f(x),
\end{gathered}
$$

where $f(x) \geq f_{0}>0$. In this case $\nabla_{w} F=\nabla_{w} G=w$ and $B(x)$ is the unit matrix.

ThEOREM 2.3. Under the conditions (O1)-(O3) there exist a subsequence $u^{\varepsilon}$ and a function $u \in V$ so that $u^{\varepsilon} \rightarrow u$ in $W_{2}^{-1}\left(\Omega ; \mathbb{R}^{N}\right)$, u belongs to $W_{p, \text { loc }}^{2}\left(\Omega ; \mathbb{R}^{N}\right)$ and satisfies the variational inequality (the obstacle problem)

$$
\begin{aligned}
& u \in \stackrel{\circ}{W}_{2}^{1}\left(\Omega ; \mathbb{R}^{N}\right), \quad F(x, u(x), \nabla u(x)) \leq 0 \quad \text { in } \Omega, \\
& \langle A u, v-u\rangle \geq 0 \\
& \quad \text { for all } v \in \stackrel{\circ}{W}_{2}^{1}\left(\Omega ; \mathbb{R}^{N}\right) \text { with } F(x, v(x), \nabla v(x)) \leq 0 \text { a.e. in } \Omega .
\end{aligned}
$$

2.9. Examples. (1) The following function $F$ satisfies the conditions (F1)(F11):

$$
F(x, w, p)=[C(x) p \cdot p+D(x) w \cdot w]^{\left(1+\alpha_{2}\right) / 2}-g(x),
$$

where the $(N n \times N n)$-matrix $C(x)=\left(c_{k s}^{l m}(x)\right)$ and $(N \times N)$-matrix $D(x)=$ $\left(d^{l m}(x)\right)$ are real and symmetric, and the elements $c_{k s}^{l m}(x), d^{l m}(x)(1 \leq l, m \leq N$; $1 \leq k, s \leq n)$ and the function $g(x)$ belong to the class $C^{2}(\bar{\Omega})$; moreover, we assume $g(x)>0$ in $\bar{\Omega}$ and

$$
\begin{gathered}
c_{0}|\eta|^{2} \leq C(x) \eta \cdot \eta=\sum_{l, m=1}^{N} \sum_{k, s=1}^{n} c_{k s}^{l m}(x) \eta_{k}^{l} \eta_{s}^{m} \leq c_{1}|\eta|^{2} \quad\left(\forall \eta \in \mathbb{R}^{N n}\right), \\
d_{0}|\zeta|^{2} \leq D(x) \zeta \cdot \zeta=\sum_{m, l=1}^{N} d^{l m}(x) \zeta^{l} \zeta^{m} \leq d_{1}|\zeta|^{2} \quad\left(\forall \zeta \in \mathbb{R}^{N}\right),
\end{gathered}
$$

where $c_{0}, c_{1}, d_{1}=$ const $>0, d_{0} \geq 0$.

(2) The conditions (F1)-(F11) are also valid for the function

$$
\begin{aligned}
F(x, w, p)= & {\left[\sum_{l=1}^{N} \sum_{k=1}^{n} f_{k}^{l}(x)\left(p_{k}^{l}\right)^{2}\right]^{\left(1+\alpha_{2}\right) / 2} } \\
& +\sum_{l=1}^{N} \sum_{k=1}^{n} g_{k}^{l}(x, w)\left|p_{k}^{l}\right|^{1+\gamma_{k}^{l}}-g(x),
\end{aligned}
$$


where $f_{k}^{l}, g \in C^{2}(\bar{\Omega}), g_{k}^{l} \in C^{2}\left(\bar{\Omega} \times \mathbb{R}^{N}\right)$, the functions $g_{k}^{l}(x, w)$ are convex with respect to $w \in \mathbb{R}^{N}$, and for $x \in \bar{\Omega}$

$$
g(x)>0, \quad g_{k}^{l}(x, w) \geq 0, \quad g_{k}^{l}(x, 0)=0 ;
$$

moreover, we assume

$$
\begin{gathered}
0<f_{0} \leq \min _{l, k} \min _{\bar{\Omega}} f_{k}^{l}(x) \leq \max _{l, k} \max _{\bar{\Omega}} f_{k}^{l}(x)=f_{1}, \\
0 \leq \min _{l, k} \gamma_{k}^{l} \leq \max _{l, k} \gamma_{k}^{l}=\gamma_{0}<\alpha_{2} .
\end{gathered}
$$

2.8. R e $m$ ar $\mathrm{k}$. The assumption (2.4) is valid if the matrix $B(x)$ or the function $F$ has a simple form. Namely, if $B(x)$ is the unit $(N \times N)$-matrix, then (2.4) holds for any convex function $F$ with properties (F1)-(F11). (2.4) is also true for any positive-definite matrix $B(x)$ and function $F$ such that the sets $\{(w, p)$ : $F(x, w, p) \leq 0\}$ are balls in $\mathbb{R}^{N} \times \mathbb{R}^{N n}$. In the general case, (2.4) is valid under some additional conditions. So, the function $F$ from the first example satisfies (2.4) if

$$
\begin{gathered}
c_{k s}^{l m}(x)=0 \quad \text { if } k \neq s, \\
B(x) C_{k}(x)=C_{k}(x) B(x), \quad 1 \leq k \leq n .
\end{gathered}
$$

3. Sketch of the proof. We keep the notation of Section 2 and assume that the conditions of Theorem 2.1 are satisfied. We first deduce some estimates for the solutions $u^{\varepsilon}$ of the penalty problem. For the sake of simplicity we often omit the index $\varepsilon$. By $C, C_{1}, C_{2}$ etc. we denote different constants not depending on $\varepsilon$.

3.1. The estimate for $\left|u^{\varepsilon}\right|_{\bar{\Omega}}$. Using the standard argument (see, e.g., $[13$, Ch. 8 , $\S 5])$ it is easy to derive the estimate $\left|u^{\varepsilon}\right|_{\bar{\Omega}} \leq C$. One has to consider the function $W(x)=\left|u^{\varepsilon}(x)\right|^{2}$ at its maximum point and use conditions (A3), (G2). Therefore we further assume that the functions $\mu_{i}\left(\left|u^{\varepsilon}(x)\right|\right)$ are bounded above by constants $\mu_{i}, i=2, \ldots, 7$, independent of $\varepsilon$ and $x \in \bar{\Omega}$. We also assume that $\nu_{3}\left(\left|u^{\varepsilon}(x)\right|\right) \geq$ $\nu_{3}=$ const $>0$ in (F5) and $R_{2}\left(\left|u^{\varepsilon}\right|\right) \leq R_{2}<\infty$.

3.2. The estimate for $\left\|\nabla u^{\varepsilon}\right\|_{L_{2}(\Omega)}$. Using (PP1) and (L2) we can write the integral identity

$$
\begin{aligned}
& \sum_{r=1}^{N} \int_{\Omega} \sum_{i, j=1}^{n}\left(a_{i j}(x) u_{x_{i}}^{r} v_{x_{j}}^{r}+\frac{\partial a_{i j}}{\partial x_{j}} u_{x_{i}}^{r} v^{r}-a^{r}(x, u, \nabla u) v^{r}\right) d x \\
&=-\int_{\Omega} \beta(F(x, u, \nabla u)) \sum_{r, m=1}^{N} b^{r m}(x) u^{m} v^{r} d x \quad\left(\forall v \in \stackrel{\circ}{W}_{2}^{1}(\Omega)\right) .
\end{aligned}
$$

The right-hand side is non-positive for any vector-function $v(x)=u(x) \Phi(x)$, where $\Phi(x) \geq 0$ is a scalar function. Proceeding as in [13, Ch. 8, §3] we obtain

$$
\left\|\nabla u^{\varepsilon}\right\|_{L_{2}(\Omega)}=\|u\|_{W_{2}^{1}(\Omega)} \leq C .
$$


3.3. The estimate for $\left|\nabla u^{\varepsilon}\right|_{\Omega^{\prime}}$. We denote by $\Omega^{\prime}$ a subdomain of $\Omega$ such that $\bar{\Omega}^{\prime} \subset \Omega$ and by $\xi$ a cut-off function for $\Omega^{\prime}$. Define $W(x)=\xi^{4} F(x, u(x), \nabla u(x))$ and evaluate $W\left(x_{0}\right)=\max _{x \in \bar{\Omega}} W(x)$. It is sufficient to consider the case $F\left(x_{0}, u\left(x_{0}\right), \nabla u\left(x_{0}\right)\right)>0,\left|\nabla u\left(x_{0}\right)\right|>R_{2}$ (see $\left.(\mathrm{F} 8)\right)$. We denote by $\widetilde{C}$ constants which do not depend on $\varepsilon$, but may depend on $\operatorname{dist}\left(\bar{\Omega}^{\prime}, \partial \Omega\right)$. At $x_{0}$ we have

$$
\begin{gathered}
W_{x_{i}}=\xi^{4} \frac{d F}{d x_{i}}+\left(\xi^{4}\right)_{x_{i}} F=0 \quad(i=1, \ldots, n), \\
0 \geq \sum_{i, j=1}^{n} a_{i j} W_{x_{i} x_{j}}=I_{1}+I_{2}+I_{3}+I_{4},
\end{gathered}
$$

where

$$
\begin{aligned}
I_{1}= & \sum_{i, j=1}^{n} \xi^{4} a_{i j}\left[\sum_{m, l=1}^{N} \frac{\partial^{2} F}{\partial w^{m} \partial w^{l}} \frac{\partial u^{m}}{\partial x_{i}} \frac{\partial u^{l}}{\partial x_{j}}+2 \sum_{m, l=1}^{N} \sum_{k=1}^{n} \frac{\partial^{2} F}{\partial w^{m} \partial p_{k}^{l}} \frac{\partial u^{m}}{\partial x_{i}} \frac{\partial^{2} u^{l}}{\partial x_{k} \partial x_{j}}\right. \\
& \left.+\sum_{m, l=1}^{N} \sum_{k, s=1}^{n} \frac{\partial^{2} F}{\partial p_{k}^{l} \partial p_{s}^{m}} \frac{\partial^{2} u^{l}}{\partial x_{k} \partial x_{i}} \frac{\partial^{2} u^{m}}{\partial x_{s} \partial x_{j}}\right], \\
I_{2}= & \sum_{i, j=1}^{n} \xi^{4}\left\{\sum_{l=1}^{N} \frac{\partial F}{\partial w^{l}} a_{i j} \frac{\partial^{2} u^{l}}{\partial x_{i} \partial x_{j}}+\sum_{l=1}^{N} \sum_{k=1}^{n} \frac{\partial F}{\partial p_{k}^{l}} a_{i j} \frac{\partial^{3} u^{l}}{\partial x_{k} \partial x_{i} \partial x_{j}}\right\}, \\
I_{3}= & \sum_{i, j=1}^{n} \xi^{4}\left\{a_{i j} \frac{\partial^{2} F}{\partial x_{i} \partial x_{j}}+2 \sum_{l=1}^{N} a_{i j} \frac{\partial^{2} F}{\partial x_{i} \partial w^{l}} \frac{\partial u^{l}}{\partial x_{j}}+2 \sum_{l=1}^{N} \sum_{k=1}^{n} a_{i j} \frac{\partial^{2} F}{\partial x_{i} \partial p_{k}^{l}} \frac{\partial^{2} u^{l}}{\partial x_{k} \partial x_{j}}\right\}, \\
I_{4}= & \sum_{i, j=1}^{n}\left[a_{i j}\left(\xi^{4}\right)_{x_{i} x_{j}} F+a_{i j}\left(\xi^{4}\right)_{x_{i}}\left[\frac{\partial F}{\partial x_{j}}+\frac{\partial F}{\partial w^{l}} \frac{\partial u^{l}}{\partial x_{j}}+\frac{\partial F}{\partial p_{k}^{l}} \frac{\partial^{2} u^{l}}{\partial x_{k} \partial x_{j}}\right]\right] .
\end{aligned}
$$

Represent $I_{1}$ in the form

$$
\sum_{r, t=1}^{N(n+1)} \sum_{i, j=1}^{n} a_{i j}\left(x_{0}\right) d^{r t}\left(x_{0}\right) q_{i}^{r} q_{j}^{t},
$$

where $d^{r t}\left(x_{0}\right)$ is the $N(n+1) \times N(n+1)$-matrix of the second derivatives of $F(x, w, p)$ with respect to $(w, p) \in \mathbb{R}^{N} \times \mathbb{R}^{n N}$ at $\left(x_{0}, u\left(x_{0}\right), \nabla u\left(x_{0}\right)\right)$. By $q=\left(q_{i}^{r}\right)$ we denote the $N n(n+1)$-dimensional vector with components $q=\left(q_{1}, \ldots, q_{n}\right)$, $q_{i}=\left(\zeta_{i}, \eta_{i}\right)$, where

$\zeta_{i}=\left(u_{x_{i}}^{1}, \ldots, u_{x_{i}}^{N}\right) \in \mathbb{R}^{N}, \quad \eta_{i}=\left(u_{x_{i} x_{1}}^{1}, \ldots, u_{x_{i} x_{n}}^{1} ; \ldots ; u_{x_{i} x_{1}}^{N}, \ldots, u_{x_{i} x_{n}}^{N}\right) \in \mathbb{R}^{N n}$.

Since the matrices $a_{i j}\left(x_{0}\right)$ and $d^{r t}\left(x_{0}\right)$ are positive-definite (see (A2) and (F5)), we obtain

$$
\begin{aligned}
\sum_{r, t=1}^{N(n+1)} \sum_{i, j=1}^{n} a_{i j} d^{r t} q_{i}^{r} q_{j}^{t} & \geq \nu_{1} \nu_{3}\left[1+|\nabla u|^{2}\right]^{\left(\alpha_{2}-1\right) / 2} \sum_{i=1}^{n}\left(\left|\eta_{i}\right|^{2}+\left|\zeta_{i}\right|^{2}\right) \\
& \geq \nu_{0}\left(1+|\nabla u|^{2}\right)^{\left(\alpha_{2}-1\right) / 2}\left|u_{x x}\right|^{2}
\end{aligned}
$$


where $\nu_{0}=\nu_{1} \nu_{3}>0$. Thus,

$$
I_{1} \geq \nu_{0}\left(1+|\nabla u|^{2}\right)^{\left(\alpha_{2}-1\right) / 2}\left|u_{x x}\right|^{2} \xi^{4} .
$$

In $I_{2}$, there are terms with third order derivatives of $u$. Therefore we differentiate the $l$ th equation of the system (PP1) with respect to $x_{k}$, multiply the result by $\partial F / \partial p_{k}^{l}$ and sum over $k, l$ to obtain

$$
\begin{aligned}
& \sum_{i, j=1}^{n} \sum_{l=1}^{N} \sum_{k=1}^{n} {\left[a_{i j} u_{x_{i} x_{j} x_{k}}^{l}+\left(a_{i j}\right)_{x_{k}} u_{x_{i} x_{j}}^{l}+\frac{d a^{l}(x, u, \nabla u)}{d x_{k}}\right] \frac{\partial F}{\partial p_{k}^{l}} } \\
&=\sum_{i, j=1}^{n} \sum_{l=1}^{N} \sum_{k=1}^{n}\left[\beta^{\prime}(F) \frac{d F}{d x_{k}} \sum_{r=1}^{N} b^{l r} u^{r}+\beta(F) \sum_{r=1}^{N}\left(b_{x_{k}}^{l r} u^{r}+b^{l r} u_{x_{k}}^{r}\right)\right] \frac{\partial F}{\partial p_{k}^{l}} .
\end{aligned}
$$

Using the $l$ th equation of (PP1) to express $a_{i j} u_{x_{i} x_{j}}^{l}$ we can write $I_{2}-J$

$$
=\xi^{4}\left[\sum_{i, j=1}^{n} \sum_{l=1}^{N} \sum_{k=1}^{n}\left(\frac{\partial F}{\partial w^{l}} a^{l}(x, u, \nabla u)-\frac{\partial F}{\partial p_{k}^{l}}\left(a_{i j}\right)_{x_{k}} u_{x_{i} x_{j}}^{l}-\frac{\partial F}{\partial p_{k}^{l}} \frac{d a^{l}(x, u, \nabla u)}{d x_{k}}\right)\right],
$$

where

$$
\begin{aligned}
J= & \xi^{4} \sum_{l, r=1}^{N}\left[\frac{\partial F}{\partial w^{l}} b^{l r} u^{r}+\sum_{k=1}^{n} \frac{\partial F}{\partial p_{k}^{l}} b^{l r} u_{x_{k}}^{r}\right] \beta(F) \\
& +\xi^{4} \beta^{\prime}(F) \sum_{l, r=1}^{N} \sum_{k=1}^{n} \frac{\partial F}{\partial p_{k}^{l}} \frac{d F}{d x_{k}} b^{l r} u^{r}+\xi^{4} \beta(F) \sum_{l, r=1}^{N} \sum_{k=1}^{n} \frac{\partial F}{\partial p_{k}^{l}}\left(b^{l r}\right)_{x_{k}} u^{r} \\
= & J_{1}+J_{2}+J_{3} .
\end{aligned}
$$

Without loss of generality one can assume that $\varepsilon<1, F\left(x_{0}, u, \nabla u\right)>1$ and $\left|\nabla u\left(x_{0}\right)\right|$ is sufficiently large. Due to the choice of the penalty function $\beta$ we have

$$
\begin{gathered}
\beta(F)=\frac{1}{\varepsilon} \beta_{0}(F) \geq F^{1+\alpha_{0}} \geq \widetilde{\nu}_{4}|\nabla u|^{\left(1+\alpha_{2}\right)\left(1+\alpha_{0}\right)}, \\
F \beta^{\prime}(F)=\frac{1}{\varepsilon} F \beta_{0}(F)=\left(1+\alpha_{0}\right) \frac{1}{\varepsilon} F^{1+\alpha_{0}}=\left(1+\alpha_{0}\right) \beta(F) .
\end{gathered}
$$

To estimate $J_{1}$ we note that

$$
\nabla_{w} G \cdot \nabla_{w} F=\sum_{l, r=1}^{N} \frac{\partial F}{\partial w^{l}} b^{l r} w^{r}
$$

and apply the assumption (2.4) of Theorem 2.1 with $w=u\left(x_{0}\right)$ and $p=\nabla u\left(x_{0}\right)$ to obtain

$$
J_{1} \geq \xi^{4} \beta(F)\left[\nu_{5}|\nabla u|^{1+\alpha_{2}}-\mu_{7}|\nabla u|^{1+\alpha_{2}-\gamma_{2}}\right], \quad \gamma_{2}>0 .
$$

Hence for sufficiently large $\left|\nabla u\left(x_{0}\right)\right|$ we have

$$
J_{1} \geq \frac{\nu_{5}}{2} \xi^{4} \beta(F)|\nabla u|^{1+\alpha_{2}} .
$$


Further, by (3.1) we have $\xi^{4} d F / d x_{k}=-\left(\xi^{4}\right)_{x_{k}} F(k=1, \ldots, n)$. Hence by $(3.5)$

$$
\begin{aligned}
J_{2} & =-F \beta^{\prime}(F) \sum_{l, r=1}^{N} \sum_{k=1}^{n} \frac{\partial F}{\partial p_{k}^{l}} b^{l r} u^{r}\left(\xi^{4}\right)_{x_{k}} \\
& =-\left(1+\alpha_{0}\right) \beta(F) \sum_{l, r=1}^{N} \sum_{k=1}^{n} b^{l r} u^{r} \frac{\partial F}{\partial p_{k}^{l}}\left(\xi^{4}\right)_{x_{k}} \\
& \geq-C \beta(F)\left[|\nabla u|^{\alpha_{2}}+1\right] \sum_{k=1}^{n}\left|\left(\xi^{4}\right)_{x_{k}}\right| .
\end{aligned}
$$

Here we have used the inequality (F10). By analogy we deduce the bound

$$
J_{3} \geq-\xi^{4} \beta(F) C\left[|\nabla u|^{\alpha_{2}}+1\right] .
$$

It is obvious that $J_{1}+J_{3} \geq\left(\nu_{5} / 4\right) \xi^{4} \beta(F)|\nabla u|^{1+\alpha_{2}}$ for large $|\nabla u|$. Note that if

$$
\xi\left|\nabla u\left(x_{0}\right)\right| \leq C \sum_{k=1}^{n}\left|\xi_{x_{k}}\right|=\widetilde{C}_{1}
$$

then $\xi^{4}|\nabla u| \leq \widetilde{C}$. If

$$
\xi\left|\nabla u\left(x_{0}\right)\right|>C \sum_{k=1}^{n}\left|\xi_{x_{k}}\right|=\widetilde{C}_{1}
$$

we obtain by $(3.4)$

$$
J=J_{1}+J_{2}+J_{3} \geq \frac{\nu_{5}}{8} \xi^{4} \beta(F)|\nabla u|^{1+\alpha_{2}} \geq \nu_{6} \xi^{4}|\nabla u|^{\left(1+\alpha_{2}\right)\left(2+\alpha_{0}\right)} .
$$

Now, we write out the derivative $d a^{l} / d x_{k}$ and use the conditions (A5) and (A6) to get

$$
\begin{aligned}
\left|\frac{d a^{l}}{d x_{k}}\right| & =\left|\frac{\partial a^{l}}{\partial x_{k}}+\frac{\partial a^{l}}{\partial w^{l}} \frac{\partial u^{m}}{\partial x_{k}}+\sum_{l=1}^{N} \sum_{s=1}^{n} \frac{\partial a^{l}}{\partial p_{k}^{l}} \frac{\partial^{2} u^{l}}{\partial x_{k} \partial x_{s}}\right| \\
& \leq C\left[|\nabla u|^{1+\alpha_{1}}+|\nabla u|^{2+\alpha_{1}}+|\nabla u|^{\alpha_{1}}\left|u_{x x}\right|\right] .
\end{aligned}
$$

In view of (A4), (F9)-(F11), (3.7) and the assumption $\left|\nabla u\left(x_{0}\right)\right|>1$ we obtain

$$
I_{2}-J \geq-\xi^{4} C\left[|\nabla u|^{2+\alpha_{1}+\alpha_{2}}+|\nabla u|^{\alpha_{1}+\alpha_{2}}\left|u_{x x}\right|\right] .
$$

For $I_{3}$ we use (F9)-(F11) to obtain

$$
I_{3} \geq \xi^{4} C\left[|\nabla u|^{2+\alpha_{2}-\delta}+|\nabla u|^{\alpha_{2}}\left|u_{x x}\right|\right] .
$$

Thus, (3.3), (3.6), (3.8) and (3.9) imply

$$
\begin{aligned}
I_{1}+I_{2}+I_{3} \geq & \xi^{4}\left[\nu_{0}\left(1+|\nabla u|^{2}\right)^{\left(\alpha_{2}-1\right) / 2}\left|u_{x x}\right|^{2}+\frac{\nu_{6}}{2}|\nabla u|^{\left(1+\alpha_{2}\right)\left(2+\alpha_{0}\right)}\right] \\
& -C \xi^{4}\left[|\nabla u|^{2+\alpha_{1}+\alpha_{2}}+|\nabla u|^{\alpha_{1}+\alpha_{2}}\left|u_{x x}\right|\right] .
\end{aligned}
$$


Apply the Cauchy inequality to obtain

$$
|\nabla u|^{\alpha_{1}+\alpha_{2}}\left|u_{x x}\right| \leq \frac{\nu_{0}}{4}\left(1+|\nabla u|^{2}\right)^{\left(\alpha_{2}-1\right) / 2}\left|u_{x x}\right|+C|\nabla u|^{1+2 \alpha_{1}+\alpha_{2}} .
$$

By (PP3)

$$
\gamma=\left(1+\alpha_{2}\right)\left(2+\alpha_{0}\right)>\max \left\{1+2 \alpha_{1}+\alpha_{2}, 2+\alpha_{1}+\alpha_{2}\right\}
$$

Therefore for sufficiently large $\left|\nabla u\left(x_{0}\right)\right|$ we deduce from (3.10)

$$
I_{1}+I_{2}+I_{3} \geq \xi^{4}\left[\frac{\nu_{0}}{2}\left(1+|\nabla u|^{2}\right)^{\left(\alpha_{2}-1\right) / 2}\left|u_{x x}\right|^{2}+\frac{\nu_{6}}{2}|\nabla u|^{\left(1+\alpha_{2}\right)\left(2+\alpha_{0}\right)}\right] \text {. }
$$

To estimate $I_{4}$ we note that

$$
\left|a_{i j}\left(\xi^{4}\right)_{x_{i} x_{j}} F\right| \leq \xi^{2} F\left|12 a_{i j} \xi_{x_{i}} \xi_{x_{j}}+4 \xi a_{i j} \xi_{x_{i} x_{j}}\right| \leq \xi^{2} \widetilde{C}|\nabla u| .
$$

Taking into account the last inequality and (F9)-(F11) we obtain for sufficiently large $\left|\nabla u\left(x_{0}\right)\right|$

$$
I_{4} \geq-\widetilde{C} \xi^{2}|\nabla u|-\widetilde{C} \xi^{3}\left(|\nabla u|^{2+\alpha_{2}-\delta}+|\nabla u|^{\alpha_{2}}\left|u_{x x}\right|\right) .
$$

By the Cauchy inequality

$$
\xi^{3}|\nabla u|^{\alpha_{2}}\left|u_{x x}\right| \leq \frac{\nu_{0}}{4} \xi^{4}\left(1+|\nabla u|^{2}\right)^{\left(\alpha_{2}-1\right) / 2}\left|u_{x x}\right|^{2}+C \xi^{2}|\nabla u|^{1+\alpha_{2}}
$$

therefore,

$$
I_{4} \geq-\frac{\nu_{0}}{4} \xi^{4}\left(1+|\nabla u|^{2}\right)^{\left(\alpha_{2}-1\right) / 2}\left|u_{x x}\right|^{2}-\widetilde{C}_{0} \xi^{2}|\nabla u|^{2+\alpha_{2}-\delta},
$$

and consequently,

$$
\sum_{i=1}^{4} I_{i} \geq \xi^{2}|\nabla u|^{2+\alpha_{2}-\delta}\left[\xi^{2} \frac{\nu_{6}}{4}|\nabla u|^{\gamma}-\widetilde{C}_{0}\right] .
$$

Now we have two possibilities: 1) $\xi^{2}\left|\nabla u\left(x_{0}\right)\right|^{\gamma} \leq 8 \widetilde{C}_{0} / \nu_{6}$ and by (F9) we have $\left.W\left(x_{0}\right) \leq \widetilde{C} ; 2\right) \sum_{i=1}^{4} I_{i} \geq \xi\left(\nu_{6} / 8\right)|\nabla u|^{\left(2+\alpha_{0}\right)\left(1+\alpha_{2}\right)}>0$; in this case by (3.2) the last inequality is not true. holds.

Thus, for any subdomain $\Omega^{\prime}$ with $\bar{\Omega}^{\prime} \subset \Omega$, the estimate $\max _{x \in \bar{\Omega}^{\prime}}\left|\nabla u^{\varepsilon}(x)\right| \leq \widetilde{C}$

3.4. The existence of the limit function $u$. Because of the estimates deduced above one can extract a subsequence from $\left\{u^{\varepsilon}\right\}$ (which we denote again by $u^{\varepsilon}$ ) such that $u^{\varepsilon l}$ converges weakly in $\stackrel{\circ}{W}_{2}^{1}(\Omega)$ to $u^{l}(l=1, \ldots, N)$. Moreover, $u^{l} \in$ $W_{\infty, \text { loc }}^{1}(\Omega)(l=1, \ldots, N)$ and, because of the imbedding theorems, $u^{\varepsilon l} \rightarrow u^{l}$ uniformly on compact sets $\bar{\Omega}^{\prime}$ with $\bar{\Omega}^{\prime} \subset \Omega$.

3.5. The estimate for $\left.\xi^{2} \beta_{\varepsilon}\left(F\left(x, u^{\varepsilon}, \nabla u^{\varepsilon}\right)\right)\right|_{\Omega_{0}^{\prime}}$. Let $\xi \in C_{0}^{\infty}(\Omega)$, supp $\xi \subset \Omega_{u}=$ $\{x \in \Omega: u(x) \neq 0\}$. Since $u$ is continuous in $\Omega$, there exists a constant $\delta>0$ such that $(\forall x \in \operatorname{supp} \xi)|u(x)|>\delta$. Since $u^{\varepsilon} \rightarrow u$ uniformly on $\bar{\Omega}^{\prime}$, we have $\left|u^{\varepsilon}(x)\right|>$ $\delta / 2$ for sufficiently small $\varepsilon$. Suppose that $W(x)=\xi^{2} \beta(F)$ has its maximum at $x_{0} \in$ $\operatorname{supp} \xi$. Now we show that $W\left(x_{0}\right)$ is bounded above by a constant which depends 
on $\delta$ and $\operatorname{dist}(\operatorname{supp} \xi, \partial \Omega)$, but does not depend on $\varepsilon$. Assume that $W\left(x_{0}\right) \neq 0$. At $x_{0}$ we have

$$
\begin{gathered}
W_{x_{i}}=\left(\xi^{2}\right)_{x_{i}} \beta(F)+\xi^{2} \frac{d \beta}{d x_{i}}=0 \quad(i=1, \ldots, n), \\
0 \geq \sum_{i, j=1}^{n} a_{i j} W_{x_{i} x_{j}}=\sum_{i, j=1}^{n}\left[a_{i j}\left(\xi^{2}\right)_{x_{i} x_{j}} \beta+2 a_{i j}\left(\xi^{2}\right)_{x_{i}} \frac{d \beta}{d x_{j}}+a_{i j} \xi^{2} \frac{d^{2} \beta}{d x_{i} d x_{j}}\right] .
\end{gathered}
$$

Since $\beta(0)=0$ and $\beta(\tau)$ is a convex function, we have $\beta(\tau) \leq \tau \beta^{\prime}(\tau)$. Substituting, in $(3.13), F \beta^{\prime}(F)$ for $\beta(F)$ we obtain the lower bound $-\widetilde{C} \beta^{\prime}$ for the first summand of (3.13). Writing out the expression for the derivative $d \beta / d x_{i}$ and taking into account the estimates for $F$ and its partial derivatives, we obtain

$$
\begin{aligned}
\sum_{i, j=1}^{n} 2 a_{i j}\left(\xi^{2}\right)_{x_{i}} \frac{d \beta}{d x_{j}} & =\sum_{i, j=1}^{n} 2 a_{i j}\left(\xi^{2}\right)_{x_{i}} \beta^{\prime}\left[\frac{\partial F}{\partial x_{j}}+\sum_{m=1}^{N} \frac{\partial F}{\partial w^{m}} u_{x_{j}}^{m}+\sum_{l=1}^{N} \sum_{k=1}^{n} \frac{\partial F}{\partial p_{k}^{l}} u_{x_{k} x_{j}}^{l}\right] \\
& \geq \beta^{\prime}\left[-\widetilde{C}-\widetilde{C} \xi\left|u_{x x}\right|\right] .
\end{aligned}
$$

Since $\beta^{\prime \prime} \geq 0$ and the matrix $a_{i j}$ is positive-definite, the third summand in (3.13) is bounded below by $\beta^{\prime} \xi^{2} a_{i j} d^{2} F / d x_{i} d x_{j}$. Thus, (3.13) implies

$$
0 \geq \sum_{i, j=1}^{n} a_{i j} W_{x_{i} x_{j}} \geq \beta^{\prime}\left[-\widetilde{C}-\widetilde{C} \xi\left|u_{x x}\right|\right]+\beta^{\prime} \xi^{2} \sum_{i, j=1}^{n} a_{i j} \frac{d^{2} F}{d x_{i} d x_{j}} .
$$

We estimate the last term in (3.14) as follows:

$$
\begin{aligned}
& \beta^{\prime} \xi^{2} \sum_{i, j=1}^{n} a_{i j} \frac{d^{2} F}{d x_{i} d x_{j}}=\beta^{\prime} \xi^{2} \sum_{i, j=1}^{n} a_{i j}\left[\frac{\partial^{2} F}{\partial x_{i} \partial x_{j}}+\sum_{m, l=1}^{N} \frac{\partial^{2} F}{\partial w^{m} \partial w^{l}} u_{x_{i}}^{m} u_{x_{j}}^{l}\right. \\
& \quad+\sum_{l, m=1}^{N} \sum_{k, s=1}^{n} \frac{\partial^{2} F}{\partial p_{k}^{l} \partial p_{s}^{m}} u_{x_{k} x_{i}}^{l} u_{x_{s} x_{j}}^{m}+\sum_{m=1}^{N} \frac{\partial^{2} F}{\partial x_{i} \partial w^{m}} u_{x_{j}}^{m} \\
& \left.\quad+\sum_{l, m=1}^{N} \sum_{k=1}^{n} \frac{\partial^{2} F}{\partial w^{m} \partial p_{k}^{l}} u_{x_{i}}^{m} u_{x_{k} x_{j}}^{l}+\sum_{m=1}^{N} \frac{\partial F}{\partial w^{m}} u_{x_{i} x_{j}}^{m}+\sum_{l=1}^{N} \sum_{k=1}^{n} \frac{\partial F}{\partial p_{k}^{l}} u_{x_{k} x_{i} x_{j}}^{l}\right] \\
& \geq \beta^{\prime} \xi^{2}\left[\nu_{0}\left|u_{x x}\right|^{2}-\widetilde{C}\left|u_{x x}\right|-\widetilde{C}+\sum_{i, j=1}^{n} \sum_{m=1}^{N} \frac{\partial F}{\partial w^{m}} a_{i j} u_{x_{i} x_{j}}^{m}\right. \\
& \left.\quad+\sum_{i, j=1}^{n} \sum_{l=1}^{N} \sum_{k=1}^{n} \frac{\partial F}{\partial p_{k}^{l}} a_{i j} u_{x_{k} x_{i} x_{j}}^{l}\right] .
\end{aligned}
$$

Here $\nu_{0} \leq \nu_{1} \nu_{3}\left(1+\left|\nabla u^{\varepsilon}\right|^{2}\right)^{\left(\alpha_{2}-1\right) / 2}$. Because of the estimate from 3.3 we can assume that $\nu_{0}>0$ does not depend on $\varepsilon$. Now, we differentiate the system 
(PP1) with respect to $x_{k}$ and sum over $k$. By (A5), (A6) and (2.4) we obtain

$$
\begin{aligned}
0 \geq & \beta^{\prime}(F) \xi^{2}\left[\nu_{0}\left|u_{x x}\right|^{2}-\widetilde{C}\left|u_{x x}\right|-\widetilde{C}\right] \\
& +\beta^{\prime}(F) \sum_{l=1}^{N} \sum_{k=1}^{n} \frac{\partial F}{\partial p_{k}^{l}}\left(\xi^{2} \frac{d \beta}{d x_{k}}\right) \sum_{s=1}^{N} b^{l s} u^{s} .
\end{aligned}
$$

By (3.12) the last summand in (3.16) can be rewritten as follows:

$$
\begin{aligned}
\beta^{\prime}(F) \sum_{l=1}^{N} \sum_{k=1}^{n} \frac{\partial F}{\partial p_{k}^{l}}\left(\xi^{2} \frac{d \beta}{d x_{k}}\right) \sum_{s=1}^{N} b^{l s} u^{s} \\
\quad=-\beta^{\prime}(F) \sum_{l=1}^{N} \sum_{k=1}^{n} \frac{\partial F}{\partial p_{k}^{l}}\left(a_{i j} u_{x_{i} x_{j}}^{l}+a^{l}\right)\left(2 \xi \xi_{x_{k}}\right) \geq-\beta^{\prime}(F)\left[\widetilde{C} \xi\left|u_{x x}\right|+\widetilde{C}\right] .
\end{aligned}
$$

Thus, it follows from (3.14) and (3.16) that

$$
0 \geq \sum_{i, j=1}^{n} a_{i j} W_{x_{i} x_{j}} \geq \beta^{\prime}(F)\left[\nu_{0} \xi^{2}\left|u_{x x}\right|^{2}-\widetilde{C} \xi\left|u_{x x}\right|-\widetilde{C}\right] .
$$

We apply the Cauchy inequality to obtain

$$
0 \geq \sum_{i, j=1}^{n} a_{i j} W_{x_{i} x_{j}} \geq \beta^{\prime}(F)\left[\nu_{0} \xi^{2}\left|u_{x x}\right|^{2}-\widetilde{C}\right] .
$$

Recalling our assumption $W\left(x_{0}\right) \neq 0$ (see 3.5) and, consequently, $\beta^{\prime}(F)>0$, we conclude that $\xi^{2}\left|u_{x x}\right| \leq \widetilde{C}$ at $x_{0}$. Hence

$$
\xi^{4} \beta^{2}\left|u^{\varepsilon}\right|^{2}=\xi^{4}\left|L u^{\varepsilon}\right|^{2}=\xi^{4} \sum_{i, j=1}^{n} \sum_{l=1}^{N}\left(a_{i j} u_{x_{i} x_{j}}^{\varepsilon l}+a^{l}\right)^{2} \leq \widetilde{C} .
$$

Since $\left|u^{\varepsilon}\left(x_{0}\right)\right|>\delta / 2$, the desired estimate is obtained.

3.6. Proof of Theorem 2.1. Because of the estimates obtained and the imbedding theorems there exists a subsequence $\left\{u^{\varepsilon}\right\}$ such that

$$
\begin{gathered}
u^{\varepsilon l} \rightarrow u^{l} \quad \text { uniformly in } \bar{\Omega}^{\prime} \subset \Omega, \\
\nabla u^{\varepsilon l} \rightarrow \nabla u^{l} \quad \text { uniformly in } \bar{\Omega}_{u}^{\prime} \subset \Omega_{u}, \\
u_{x x}^{\varepsilon l} \rightarrow u_{x x}^{l} \quad \text { weakly in } L_{q}\left(\Omega_{u}^{\prime}\right)(1<q<\infty),
\end{gathered}
$$

where $u=\left(u^{1}, \ldots, u^{N}\right)$ is the limit function from 3.4. Moreover,

$$
u \in C^{0,1}\left(\Omega ; \mathbb{R}^{N}\right) \cap C^{1}\left(\Omega_{u} ; \mathbb{R}^{N}\right) \cap W_{2, \text { loc }}^{2}\left(\Omega_{u} ; \mathbb{R}^{N}\right),
$$

i.e., $u$ satisfies (QV1). By (3.17) and (3.18) we have $\beta_{0}\left(F\left(x, u^{\varepsilon}, \nabla u^{\varepsilon}\right)\right) \rightarrow$ $\beta_{0}(F(x, u, \nabla u))$ for $x \in \Omega_{u}$. Since $\beta_{\varepsilon}(\tau)=(1 / \varepsilon) \beta_{0}(\tau)$, the estimate from 3.5 provides $\beta_{0}(F(x, u, \nabla u))=0$. Hence $F(x, u, \nabla u) \leq 0$ for $x \in \Omega_{u}$, i.e., the inequality (QV3) is valid. To verify the quasivariational inequality (QV2) let us note that from (3.17), (3.18) we have

$$
\left\langle A u^{\varepsilon}, \eta\left(v-u^{\varepsilon}\right)\right\rangle \rightarrow\langle A u, \eta(v-u)\rangle
$$


for $\eta$ from (QV2) and $v \in \stackrel{\circ}{W}_{2}^{1}\left(\Omega ; \mathbb{R}^{N}\right)$. It follows from (PP1) that

$$
\left\langle A u^{\varepsilon}, \eta\left(v-u^{\varepsilon}\right)\right\rangle \leq 0
$$

for any $v \in \stackrel{\circ}{W}_{2}^{1}\left(\Omega ; \mathbb{R}^{N}\right)$ such that $v(x) \in M\left(x, u^{\varepsilon}(x)\right)$ a.e. in $\Omega$. Now we set $\delta=\min _{x \in \operatorname{supp} \eta}|u(x)|$. We have $\delta>0$ and $G(x, u(x)) \geq \nu_{4} \delta^{2}$ for $x \in \operatorname{supp} \eta$. Let $v$ be an arbitrary function in $\mathscr{\circ}_{2}^{1}\left(\Omega ; \mathbb{R}^{N}\right)$ such that $v(x) \in M(x, u(x))$ a.e. in $\Omega$. Define $v_{n}(x)=(1-1 / n) v(x)$. Note that $v_{n}(x) \in M(x, u(x))$ and

$$
G\left(x, v_{n}\right)=B(x) v_{n} \cdot v_{n}=\left(1-\frac{1}{n}\right)^{2} G(x, v) \leq\left(1-\frac{1}{n}\right)^{2} G(x, u) .
$$

By (3.17) we also have $G\left(x, v_{n}\right) \leq G\left(x, u^{\varepsilon}\right)$ for $x \in \operatorname{supp} \eta$ if $\varepsilon$ is sufficiently small. Hence we obtain (QV2) from (3.20) as $\varepsilon \rightarrow 0$ and $n \rightarrow \infty$. To check (QV4) one has to use (3.17) and (3.18).

3.7. Proof of Theorem 2.2. Proceeding as in [13, Ch. 8] with the help of the barrier technique one can obtain the conclusion of the theorem.

3.8. Proof of Theorem 2.3. The proof of the estimates for $\left|u^{\varepsilon}\right|_{\bar{\Omega}}$, $\left\|u^{\varepsilon}\right\|_{W_{2}^{1}(\Omega)}$ and $|\nabla u|_{\partial \Omega}$ is quite similar to that in Theorems 2.1 and 2.2. To deduce the estimate for $|\nabla u|_{\bar{\Omega}}$ consider the function $W(x)=|\nabla u|^{2}$ at its maximum point $x_{0} \in \Omega$. The inequality

$$
\begin{aligned}
0 \geq \Delta W & =2 u_{x_{k} x_{j}}^{l} u_{x_{k} x_{j}}^{l}+2 u_{x_{k}}^{l} u_{x_{k} x_{j} x_{j}}^{l} \\
& \geq\left|u_{x_{x}}\right|^{2}-2 \beta^{\prime} f_{x_{k}} u_{x_{k}}^{l} u^{l}+2 \beta^{\prime}\left(u^{l} u_{x_{k}}^{l}\right)^{2}+2 \beta|\nabla u|^{2}
\end{aligned}
$$

provides the desired estimate.

Note that $\beta_{\varepsilon}\left(F\left(x, u^{\varepsilon}\right)\right)=0$ if $\left|u^{\varepsilon}(x)\right| \leq f_{0}$ (see (O3)). Taking this into account and proceeding as in 3.5 we can establish the estimate $\beta_{\varepsilon}\left(F\left(x, u^{\varepsilon}\right)\right) \leq C$ for all $x \in \bar{\Omega}$. Arguing as in the proof of Theorem 2.1 we deduce that $F(x, u(x)) \leq 0$ in $\bar{\Omega}$ and $L u(x)=\lambda(x) u(x)$ where $\lambda(x) \geq 0$. Moreover, $\lambda(x)=0$ if $F(x, u(x))<0$. Thus, we conclude that the solution $u$ of (QV1)-(QV4) (or (U1)-(U5)) satisfies the obstacle problem of type (1.19).

\section{References}

[1] A. A. Arkhipova, Regularity of the solution of a system of variational inequalities with constraint in $\mathbb{R}^{N}$, Vestnik Leningrad. Univ. 1984 (13), 5-9 (in Russian).

[2] - Regularity of the problem with an obstacle up to the boundary for strongly elliptic operators, in: Some Applications of Functional Analysis to Problems of Mathematical Physics, Inst. Math., Siberian Branch of Acad. Sci. USSR, Novosibirsk 1988, 3-20 (in Russian).

[3] - Minimal supersolutions for the obstacle problem, Izv. Akad. Nauk SSSR 37 (1973), 1156-1185 (in Russian).

[4] A. A. Arkhipova and N. N. Ural'tseva, The regularity of solutions of variational inequalities under convex boundary constraints for a class of non-linear operators, Vestnik Leningrad. Univ. 1987 (15), 13-19 (in Russian). 
[5] L. Caffarelli, Further regularity for the Signorini problem, Comm. Partial Differential Equations 4 (1979), 1067-1075.

[6] L. C. Evans, A second order elliptic equation with gradient constraint, ibid. 4 (1979), 555-572 and 1199.

[7] A. Friedman, Variational Principles and Free-Boundary Problems, Wiley, New York 1982.

[8] S. Hildebrandt and K.-O. Widman, Variational inequalities for vector-valued functions, J. Reine Angew. Math. 309 (1979), 191-220.

[9] H. Ishii and S. Koike, Boundary regularity and uniqueness for an elliptic equation with gradient constraint, Comm. Partial Differential Equations 8 (1983), 317-346.

[10] R. J ensen, Regularity for elastic-plastic type variational inequalities, Indiana Univ. Math. J. 32 (1983), 407-423.

[11] D. Kinderlehrer, The smoothness of the solution of the boundary obstacle problem, J. Math. Pures Appl. 60 (1981), 193-212.

[12] D. Kinderlehrer and G. Stampacchia, An Introduction to Variational Inequalities and Their Applications, Acad. Press, New York 1980.

[13] O. A. Ladyzhenskaya and N. N. Ural'tseva, Linear and Quasilinear Elliptic Equations, Nauka, Moscow 1973 (in Russian).

[14] H. Lewy and G. Stampacchia, On the regularity of the solution of a variational inequality, Comm. Pure Appl. Math. 22 (1969), 153-188.

[15] J. L. Lions, Quelques méthodes de résolution des problèmes aux limites non linéaires, Dunod, Paris 1969.

[16] T. N. Rozhkovskaya, Unilateral problems with convex constraints on the gradient, in: Partial Differential Equations, Proc. S. L. Sobolev Seminar, Novosibirsk 1981, 78-85 (in Russian).

[17] - The smoothness of the solutions of the variational inequalities with gradient constraints, in: The Imbedding Theorems and Their Applications, Proc. S. L. Sobolev Seminar, Novosibirsk 1982, 128-138 (in Russian).

[18] -, On one-sided problems for non-linear operators with convex constraints on the gradient of the solution, Dokl. Akad. Nauk SSSR 268 (1983), 38-41 (in Russian). English transl. in Soviet Math. Dokl. 27 (1983).

[19] _- The regularity theorem for a unilateral problem with the convex constraints on the gradient of the solution, in: Problemy Mat. Anal. 9, Izdat. Leningrad. Univ., Leningrad 1984, 166-171; English transl. in J. Soviet Math. 35 (1) (1986).

[20] -, Unilateral problems for elliptic operators with convex constraints on the gradient of the solution, Sibirsk. Mat. Zh. 26 (3) (1985), 134-146 and 26 (5) (1985), 150-158 (in Russian).

[21] —, One-sided problems for parabolic quasilinear operators, Dokl. Akad. Nauk SSSR 290 (3) (1986), 549-552 (in Russian).

[22] -, Unilateral problems with convex constraints for quasilinear parabolic operators, Sibirsk. Mat. Zh. 29 (5) (1988), 198-211 (in Russian).

[23] G. M. Troianiello, Maximal and minimal solutions to a class of elliptic quasilinear problems, Proc. Amer. Math. Soc. 91 (1) (1984), 95-101.

[24] N. N. U ral'tseva, Hölder continuity of gradients of solutions of parabolic equations under the Signorini conditions on the boundary, Dokl. Akad. Nauk SSSR 280 (3) (1985), 563-565 (in Russian).

[25] - On the regularity of solutions of variational inequalities, Uspekhi Mat. Nauk 42 (6) (1987), 151-174 (in Russian).

[26] M. Wiegner, The $C^{1,1}$-character of solutions of second order elliptic equations with gradient constraint, Comm. Partial Differential Equations 6 (1981), 361-371.

[27] G. H. Williams, Nonlinear nonhomogeneous elliptic variational inequalities with a nonconstant gradient constraint, J. Math. Pures Appl. 60 (2) (1981), 213-226. 